annual lectures which keep alive the memory of the most famous of the Secretaries of the Institution, James Forrest, he took for his subject " The Interdependence of Abstract Science and Engineering." That was in 1893. Since then many men famous both as engineers and as men of science have delivered the James Forrest Lecture, but the happy choice of the Council in selecting Sir Alfred Ewing to give the lecture which will mark the centenary of the incorporation of the
Institution will not fail to gain general approval; his lecture on "A Century of Inventions" will be awaited with more than usual interest. In the voluminous publications of the Institution, which in themselves form a veritable engineering library, it may be there are few, if any, more valuable records than these James Forrest lectures, which have often rivalled in interest the famous presidential addresses of Sir John Rennie, Robert Stephenson, Sir William White, and others.

\title{
The Accuracy of Shortt Free Pendulum Clocks.
}

By Dr. J. JACKson and W. Bowyer.

THE period of vibration of a simple pendulum, swinging in a vacuum through the small semi-arc $\alpha$ is given by $2 \pi \sqrt{\frac{\bar{l}}{g}}\left(1+\frac{1}{16} a^{2}\right)$.

Variations in the rate of a pendulum clock are produced by variation in (1) the length of the pendulum ; (2) the arc of vibration; and variation in several factors which produce a departure from the above formula; namely: (3) air resistance; (4) elasticity of the spring; (5) interference with the free motion of the pendulum by the escapement and impulsing mechanism.

We will consider these in turn.

(l) The principal cause of variation in the length of the pendulum is change of temperature. This can be overcome by keeping the pendulum at constant temperature. Attempts have also been made with more or less success to compensate the effect by the use of metals of different coefficients of expansion so as to make the effective length of the pendulum practically independent of the temperature. But the parts of such compound pendulums may not take up changes of temperature at the same rate, and those of the grid-iron type are apt to roll. The discovery of invar has greatly simplified the temperature question. But invar is a rather unstable substance and its growth produces slow secular change in the clock rate.

(2) If the bob moves in a cycloid instead of a circle the period is independent of the amplitude. Clocks have been constructed with 'cycloidal cheeks' to guide the pendulum, but these have not proved of value. When the semi-arc of vibration is $1^{\circ}$ a change of $1^{\prime}$ in the amplitude affects the daily rate of a simple pendulum by only $0.05 \mathrm{~s}$. The change of arc in precision clocks is not great, and, moreover, as a result of the action of the suspension spring, the effect of change of arc in such clocks may easily be less than the theoretical amount given, so that this source of irregularity is not very serious.

(3) A change of 1 per cent. in the air pressure under ordinary conditions changes the daily rate of a standard type of pendulum by about $0.1 s$. This can be fairly accurately compensated in various ways, but for the most accurate clocks it is best to keep the pressure constant. For Riefler clocks this is usually about $600 \mathrm{~mm}$. of mercury, but for the Shortt clocks it is of the order of $30 \mathrm{~mm}$.

(4) The effect of the suspension spring does not appear to be very great, although various methods of supporting the pendulum have been invented. Change of elasticity with temperature and fatigue of the material from continuous bending, may produce variations of arc and so affect the rate of the clock.

(5) The most difficult problem in clock making has been in the escapement. In the usual type of clock the escapement serves a double purpose. It enables the number of vibrations to be counted, and through it the impulse is given which maintains the vibrations of the pendulum. The pendulum is in more or less continuous contact with the escape wheel, and although this is generally situated fairly near the point of suspension, it is clear that frictional forces and continuous interference by the escape wheel may easily produce considerable irregularity. in the clock rate.

At the end of 1924 the clock Shortt 3, made by the Synchronome Co., Ltd., was installed at the Royal Observatory, Greenwich, and it proved so reliable that it was introduced as standard on Jan. 1, 1925. It consists of a 'free pendulum' and a 'slave clock.' The free pendulum is made of invar swinging in an air-tight case at a pressure of about 1 inch of mercury. The free pendulum has no escapement. On a bar about one-fifth of the way from the top it carries a very light wheel about $6 \mathrm{~mm}$. in diameter. At every fifteenth swing to the left (30 seconds) a gravity lever carrying a weight of $\frac{1}{3} \mathrm{gm}$. and released by the slave clock, falls on the wheel, giving it an impulse. As this lever gets clear of the wheel a tail-piece releases mechanism which resets the impulse lever and also causes an electric contact to be made which synchronises the slave clock. An action of the synchroniser on the slave clock advances its phase by approximately $0 \cdot 004 s$. The slave clock is rated to lose about $5 s$. a day, or $0.002 s$. per $30 s$. Under these conditions the synchronising action on the slave pendulum occurs at alternate half minutes with considerable regularity. The slave clock does all the work of counting the vibrations and releasing the impulse lever. Consequently, the only interference with the free pendulum is the impulsing during a fraction of a second every thirty seconds. The great advantage which the Shortt clock has shown over all other types of clock is undoubtedly due to the relative freedom of the pendulum from mechanical interference.

This clock was started at a practically zero rate

No. 3057, VoL. 121] 
at the beginning of 1925 , but it acquired a losing rate at about $0 \cdot 048$. per day per month. By the autumn of that year the rate of change of rate had fallen to about $0.012 s$. per day per month. It remained at approximately this value until June 1926, when the clock stopped as a result of the failure of the release of the lever which resets the impulse arm of the free pendulum. This failure was caused by two steel surfaces jamming, and to avoid a recurrence of the fault a jewel was introduced at the locking surface.

The clock was restarted in July 1926, since which time its performance has been remarkable. The rate showed the previous change of about $0.012 s$. per day per month, but apart from this the going appeared nearly uniform. During October and November 1926, when special time determinations were being made for longitude purposes, the temperature was subject to unusually large fluctuations, and it appeared that there might be a temperature term of the order of $0.003 s$. per day per $1^{\circ} \mathrm{F}$. Consequently thermostatic control was introduced, and the clock rate became very steady. This continued until May 1927, when the clock error commenced to depart from the predicted value, but this was traced to an irregularity in sidereal time and not in the clock rate. For those not acquainted with the exact definition of sidereal time, we may state that sidereal time is defined by the hour angle of the first point of Aries measured westward from the meridian. The motion of the first point of Aries is for convenience divided into two parts, the uniform part called precession and the nonuniform part called nutation. The principal part of the latter is given by the formula

$$
-1.06 s \cdot \sin \Omega-0.08 s \cdot \sin 2 L \text {. }
$$

The period of the first of these terms is 18.6 years, and this term can with sufficient accuracy be expressed in the form $\alpha+\beta t+\gamma t^{2}$ for an interval of a year or two. The second term, though of much smaller amplitude, runs through its period in six months, and this term cannot be expressed in quadratic form for more than four or five months. The clock error was therefore analysed in the form $a+\beta t+\gamma t^{2}+$ nutation, and this satisfactorily explained all the observations while the temperature was approximately constant. The temperature of the clock room had been maintained near $55^{\circ} \mathrm{F}$., but in the summer of 1927 it rose to about $62^{\circ} \mathrm{F}$., and by comparison of the observed clock error with the formula, it was found that there was a temperature term of very nearly $0.0030 s$. per day per $1^{\circ} \mathrm{F}$. When this effect was allowed for, a formula was obtained which fitted all the observations from March 1927 to December within 0.1s. The formula was extrapolated backwards and found to leave residuals of about three-quarters of a second in the autumn of 1926 and to fit the observations

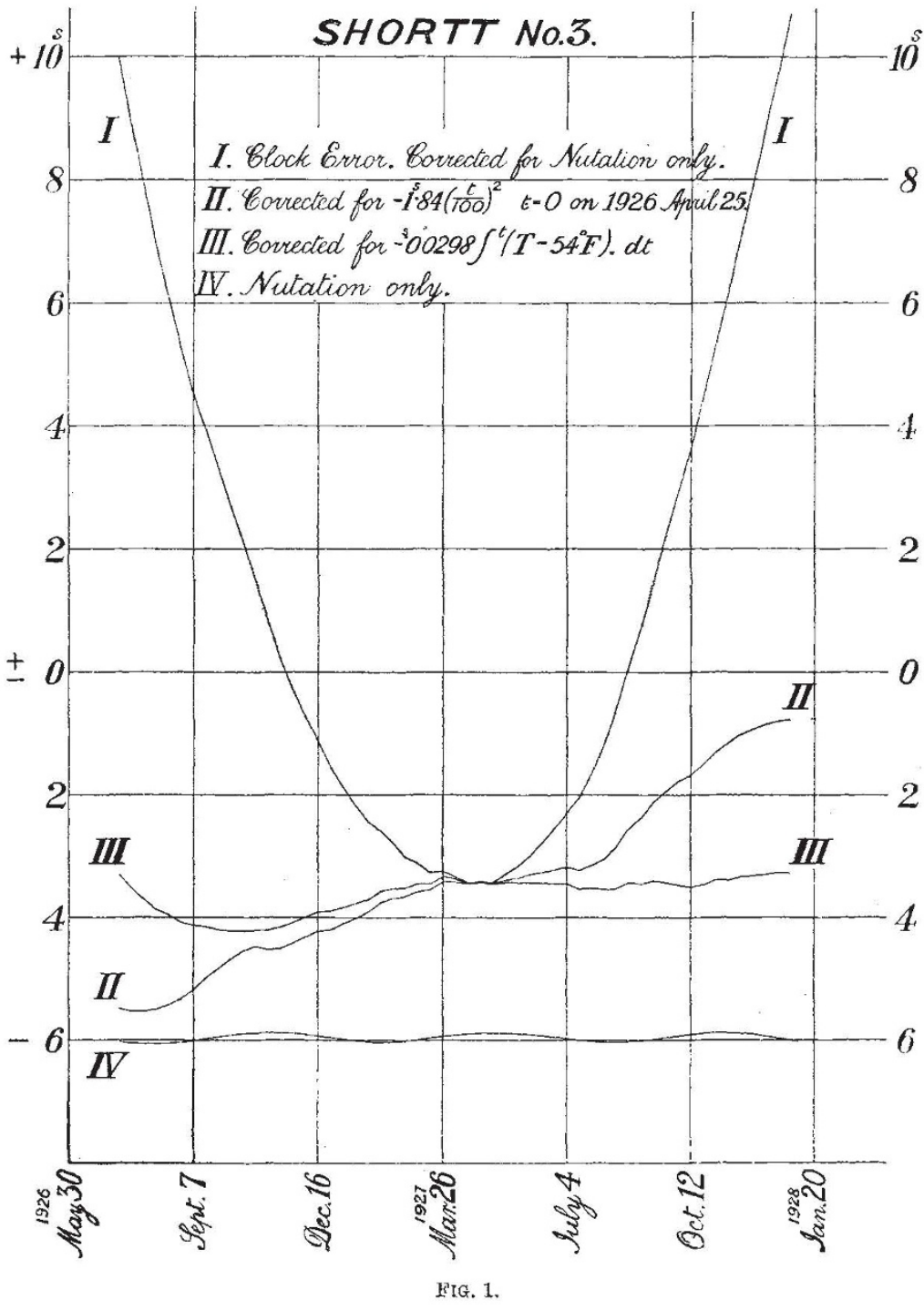

again in July 1926. Extrapolation forwards fits the observations to $0 \cdot 1 s$. at the end of March 1928.

Itshould be stated that although the temperatures were not known with great accuracy in 1926, the residuals must be due to change in $\gamma$ or some other irregularity.

The two diagrams (Figs. 1 and 2) show how closely the observed clock errors can be explained when the irregularities are allowed for. The points in the diagrams are based on curves drawn

No. 3057, VoL. 121] 
through all the errors determined with the transit circle. A more detailed comparison of the observed and computed clock errors is given in the Monthly Notices of the Royal Astronomical Society for March 1928.

The temperature term of $0.0030 s$. per day per $1^{\circ} \mathrm{F}$, or $1 \cdot 1 s$, per year per $1^{\circ} \mathrm{F}$, is much smaller than that for most high-grade clocks, but it was not expected to exist. It is said by the makers to be larger than would arise from uncertainty in the

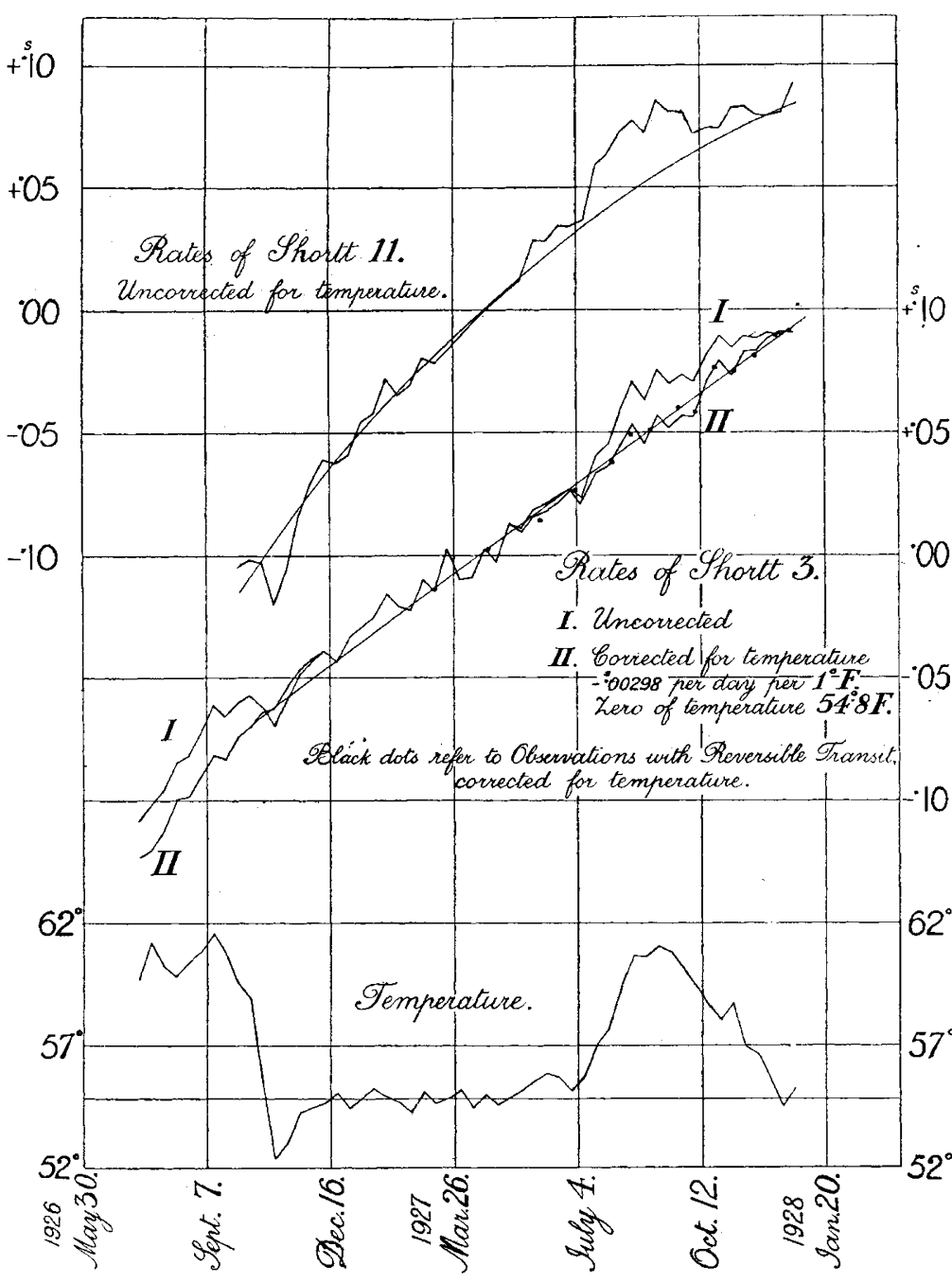

Fia. 2.

coefficients of expansion of the materials used in the construction of the pendulum. The second Shortt clock which was erected in the same clock room in May 1926 shows a temperature coefficient of similar amount.

The temperature term in the clock error is, however, of comparatively small importance. The important term for long-distance forecasting of the clock error is $\gamma t^{2}, \gamma$ being $1.84 s$. when $t$ is in units of 100 days. This term will amount to more than three minutes in 3 years. There can be little doubt that it is due to growth in the length of the pendu- lum. It is known that invar grows for years after its manufacture, and that the growth may be irregular. To explain the above coefficient the growth in the length of the pendulum $(994 \mathrm{~mm}$.) is $0.001 \mathrm{~mm}$. in 118 days. Changes in the rate of growth of the pendulum do not affect the prediction of clock errors for a month or so, but may become serious when a formula fitting the observations for a year or more is considered.

Possible changes in the rate of rotation of the earth have recently attracted the attention of astronomers. Observations of the positions of the moon, supported fairly well by observa. tions of more slowly moving bodies in the solar system, indicate that the rotation of the earth may be subject to variation amounting in extreme cases to about $1 s$. per year. One second a year is only 1 part in 30 millions, and if residuals of this order in the relative times shown by the earth and the free pendulum are demonstrated, the question will arise as to whether they are caused by :

(a) Residual secular change in the length of the pendulum.

(b) Variation of gravity.

(c) Variation in the actual rate of rotation of the earth.

(d) Seismic disturbances.

(b) and (c) may be inseparable, as changes in the earth's moment of inertia may be accompanied by changes in gravity as well as in the earth's angular velocity.

The real difficulty in the clocks is (a). It appears that the principle of their construction is such that they could be used to check the uniformity of the earth's rotation if only material stable for several years to 1 part in 100 millions could be obtained for the manufacture of the pendulums. At present the two sidereal Shortt clocks at Greenwich have been running continuously for 20 months, and a run of a few years would possibly suffice for errors of $1 s$. to accumulate in the earth's rotation, but a variation of 1 per cent. in the rate of growth of the pendulums would introduce greater irregularity in the clock error. It appears impossible to be certain that any piece of material has the required degree of stability, and until pendulums of different materials in different parts of the earth agree in supporting the motion of the moon and planets against the earth's rotation, clocks will not play an important part in checking the uniformity of the earth's rotation.

No. 3057, Vor. 121] 